\section{A review of binimetinib for the treatment of mutant cutaneous melanoma}

\author{
Peter Koelblinger ${ }^{1,2}$, Joelle Dornbierer ${ }^{1} \&$ Reinhard Dummer ${ }^{*, 1}$
}

\begin{abstract}
Although significant progress has been made in the treatment of unresectable or metastatic melanoma, at least half of all advanced melanoma patients eventually progress and pass away due to their disease. Especially patients with NRAS-mutated melanoma still face limited therapeutic options, with immunotherapy being the current treatment type of choice. Binimetinib is a selective inhibitor of MEK, a central kinase in the tumor-promoting MAPK pathway. The results of a recent Phase III trial rendered binimetinib the first targeted therapy agent to significantly improve progression-free survival in NRAS-mutated melanoma. This review will summarize the development and clinical data of binimetinib in melanoma in general and also explore the potential future role of this substance as single agent or combination therapy.
\end{abstract}

First draft submitted: 10 April 2017; Accepted for publication: 12 May 2017; Published online:

Recently, the advent of targeted therapies and immunotherapy in metastatic melanoma has significantly changed the therapeutic landscape of this devastating disease with impressive tumor responses seen after kinase inhibitor treatment along with a small proportion of patients even achieving long-term survival of 10 years or more after immunotherapy with CTLA- 4 antibodies [1-7]. Despite the availability and effectiveness of these novel treatments, the majority of patients with advanced melanoma still eventually face fatal progression of their disease, thus maintaining an unmet need for the development of further therapeutic agents.

While immunotherapy with anti-CTLA-4 or anti-PD1-antibodies in melanoma utilizes a rather universal approach harnessing the patients' own immune system, in other words, $\mathrm{T}$ cells to attack and kill aberrant melanocytic tumor cells, targeted therapies, such as BRAF- or MEK-inhibitors aim to reverse the effects of distinct oncogenic mutations that confer proliferation and survival of melanoma cells. Increasing insight into the mutational landscape of cutaneous melanoma by large-scale genomic studies - published by The Cancer Genome Atlas Network and others - has led to the definition of four distinct mutational profiles based on genetic alterations impacting the MAPK pathway in cutaneous melanoma [8,9]. Being the most frequent, BRAF-mutations (predominantly the V600E hotspot mutation) occur in $40-50 \%$ of all melanomas, while NRAS- and NF1 mutations are less common, found in approximately 20 and $15 \%$ of melanomas, respectively [8-11]. Of note, BRAF and NRAS mutations are considered to be mutually exclusive despite the recent demonstration of their co-occurrence in melanoma cells, whereas loss-of-function mutations in the tumor suppressor gene NF1 can infrequently be detected both in BRAF- and NRAS-mutated melanomas, albeit being most common in $\mathrm{BRAF}^{\mathrm{W}_{\mathrm{t}}} / \mathrm{NRAS}^{\mathrm{W}_{\mathrm{t}}}$ tumors [9,12]. Last, melanomas belonging to the so-called triple

'Department of Dermatology, University Hospital of Zurich, Zurich, Switzerland

Department of Dermatology, Paracelsus Medical University, Salzburg, Austria

*Author for correspondence: Tel.: +41 44255 25 07; Reinhard.dummer@usz.ch

\section{KEYWORDS}

- binimetinib - BRAF-

mutated melanoma $\bullet$ MAPK

pathway $\bullet$ NRAS mutations 
wild-type subgroup do not harbor any of the above listed but distinct other driver mutations and account for another $15 \%$ of melanomas [8,9]. Certain clinical characteristics - such as age or location of the primary tumor - have also been linked to the four mutational subgroups of melanoma discussed above. For example, patients with BRAF-mutated melanoma tend to be younger, while triple wild-type patients are significantly older than average in the The Cancer Genome Atlas Network cohort [8].

Mutation-specific targeted therapy has to date only been established in BRAF-mutated melanoma. In 2011, vemurafenib, a potent orally bioavailable inhibitor of the V600E-mutated BRAF kinase, was the first substance to achieve improved rates in progression-free and overall survival (OS) in BRAF-mutated advanced melanoma [1]. A few months later, similar results were reported for another BRAF inhibitor, dabrafenib [3]. Despite initial objective response rates (ORRs) of roughly $50 \%$ with these substances, treatment resistance occurs in the majority of patients after a median of 5-6 months. Resistance development is predominantly mediated via reactivation of the MAPK pathway (Figure 1) through different mechanisms [13]. To overcome development of resistance, dual inhibition of the MAPK pathway with BRAF- and MEK inhibitors was investigated subsequently and led to improvement of both response rates (RRs) and duration of response (DOR) as compared with BRAF inhibitor monotherapy $[4,6]$. However, treatment resistance remains an issue, occurring after a median duration of 11-12 months. At present, combination therapy with a BRAF- and MEK inhibitor - either $\mathrm{V}+\mathrm{C}$ or $\mathrm{D}+\mathrm{T}$, respectively - is considered standard of care in advanced BRAF-mutated melanoma and recommended as first or second line of therapy in international guidelines [14-16].

According to a recent meta-analysis, NRAS mutations occur in $18 \%$ of melanomas, most commonly in codon 61 (Q61R or Q61K) [11]. Similar to BRAF V600 mutations which constitutively activate the BRAF protein, NRAS Q61 mutations lock the NRAS protein in its active state leading to activation of the MAPK pathway and promotion of tumor cell growth and survival. As opposed to mutant BRAF, though, direct targeting of the mutant NRAS GTPase has not proven successful to date, mainly due to the high affinity of Q61 mutant NRAS to GTP which keeps the NRAS protein in its active state.
Other attempts to target mutant NRAS are summarized elsewhere in detail, but, to date, have not shown clear success in vivo [17]. Hence, indirect targeting of NRAS signaling via inhibition of molecules further downstream in the MAPK pathway, such as MEK or ERK has evolved as an alternative strategy to combat NRAS-mutant melanoma.

Binimetinib, previously known as MEK162 or ARRY-438162, is an orally bioavailable selective inhibitor of the highly homologous kinases MEK 1 and 2 and was the first substance to show clinical activity in NRAS-mutated melanoma patients in a Phase II clinical trial [18]. In parallel and subsequently, further clinical trials with binimetinib - also in BRAF-mutated melanoma - have been conducted. The present review will summarize the existing clinical data of binimetinib in melanoma as well as its pharmacokinetic (PK) and safety profile. Future possibilities of binimetinib use in advanced melanoma as a single agent or in combination with other therapeutics will also be explored.

\section{PK parameters \\ - Absorption}

Different doses of binimetinib have been investigated in multiple Phase I studies in healthy subjects and cancer patients [19]. Plasma concentrations of the different binimetinib dosing schemes evaluated are shown in Figure 2. In a Phase II clinical trial with binimetinib, PK profiling was performed on day 1 and 15 of the first therapeutic cycle. A total of 22 patients with advanced melanoma, harboring an NRAS or BRAF mutation receiving $45-\mathrm{mg}$ binimetinib two-times a day (b.i.d.) were involved in the analysis [18,19]. After oral administration, binimetinib was absorbed rapidly, with a median $\mathrm{T}_{\text {max }}$ of $1.48 \mathrm{~h}$. The between-patient reproducibility of exposure was similar on days 1 and 15 (31-40\% coefficient of variation), ruling out the nonlinearity of PKs. The PK of 45-mg binimetinib was analyzed separately for BRAF- and NRAS-mutant patients. No relevant difference between groups was detected.

The influence of food intake $30 \mathrm{~min}$ prior to drug ingestion was investigated in clinical study CMEK162A2103 [19]. Therein, a low-fat meal increased maximum plasma concentration $\left(\mathrm{C}_{\max }\right)$ of binimetinib by approximately $29 \%$ on average, while a high-fat meal decreased the estimated geometric mean $\mathrm{C}_{\max }$ by approximately $17 \%$ on average, compared with fasting 


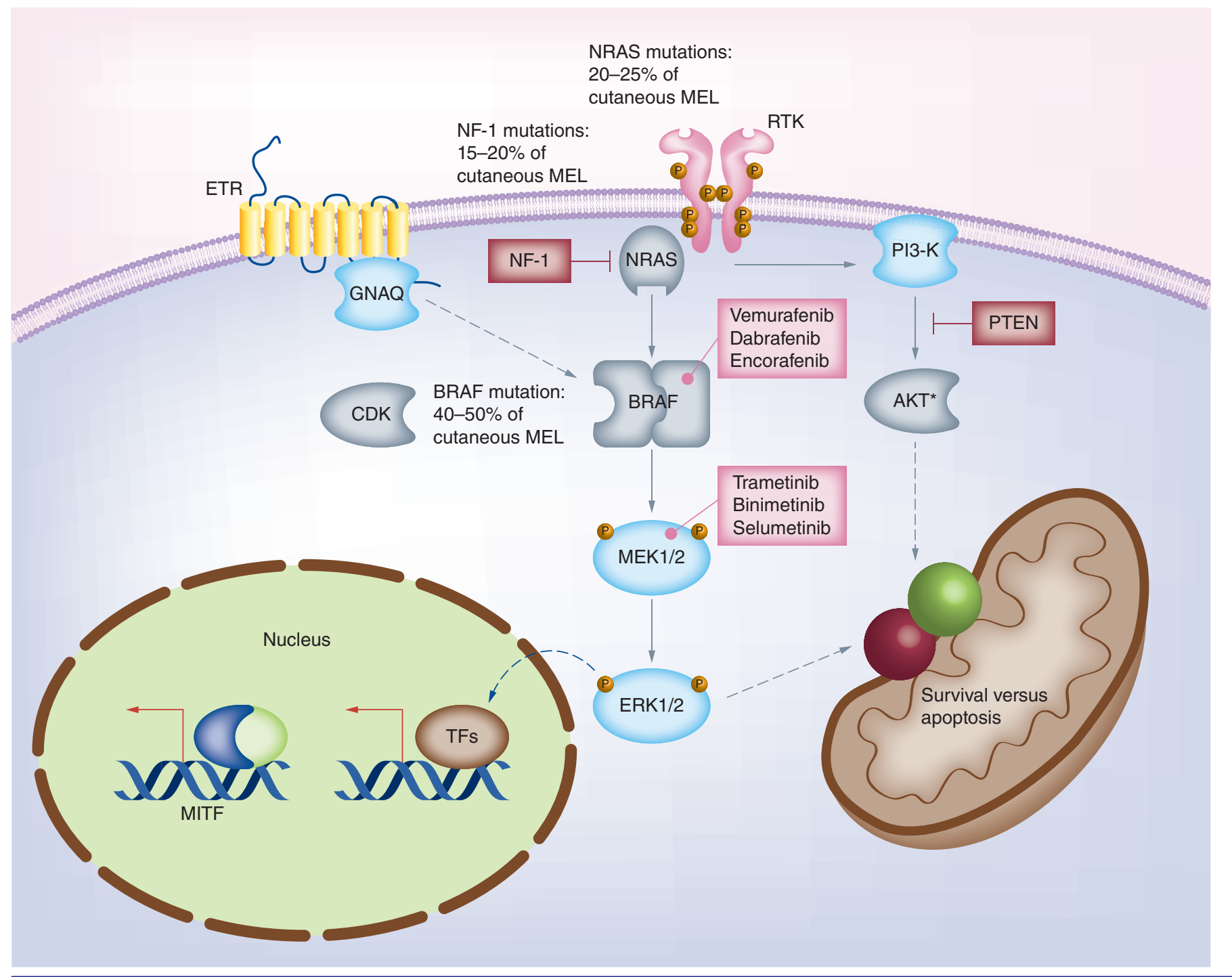

Figure 1. The MAPK pathway in melanoma - frequencies of relevant mutations and pharmacological inhibitors of BRAF and MEK.

conditions. $\left(\mathrm{T}_{\max }\right)$ was increased both after low- and high-fat food intake, suggesting that food delayed the systemic absorption of binimetinib. Conversely, the mean plasma area under the curve was not significantly affected by food intake suggesting that the total amount of absorbed binimetinib is not altered by food intake prior to drug administration.

\section{- Distribution}

The clinical study CMEK162A2102 examined the absorption, distribution, metabolism and elimination of a single oral dose of $45 \mathrm{mg}$ of ${ }^{14} \mathrm{C}$-binimetinib in six healthy male subjects [19]. The overall recovery of radioactivity in the excreta was $93.6 \pm 3.27 \%$ in these patients, pointing out that the mass balance was achieved. The mean blood plasma concentration ratio for total radioactivity was similar to the mean in vitro value for ${ }^{14} \mathrm{C}$-binimetinib (0.62 vs 0.72 ). On average, about $60 \%$ of the circulating radioactivity AUC in plasma was traceable to binimetinib.

\section{- Metabolism}

In the same absorption, distribution, metabolism and elimination study, binimetinib was shown to underlie primary biotransformation, such as glucuronidation, N-dealkylation, amide hydrolysis and loss of ethanediol from the side chain in humans. Secondary biotransformation pathways involving the primary biotransformation products included glucuronidation, oxygenation and dehydrogenation. CYP1A2 and 


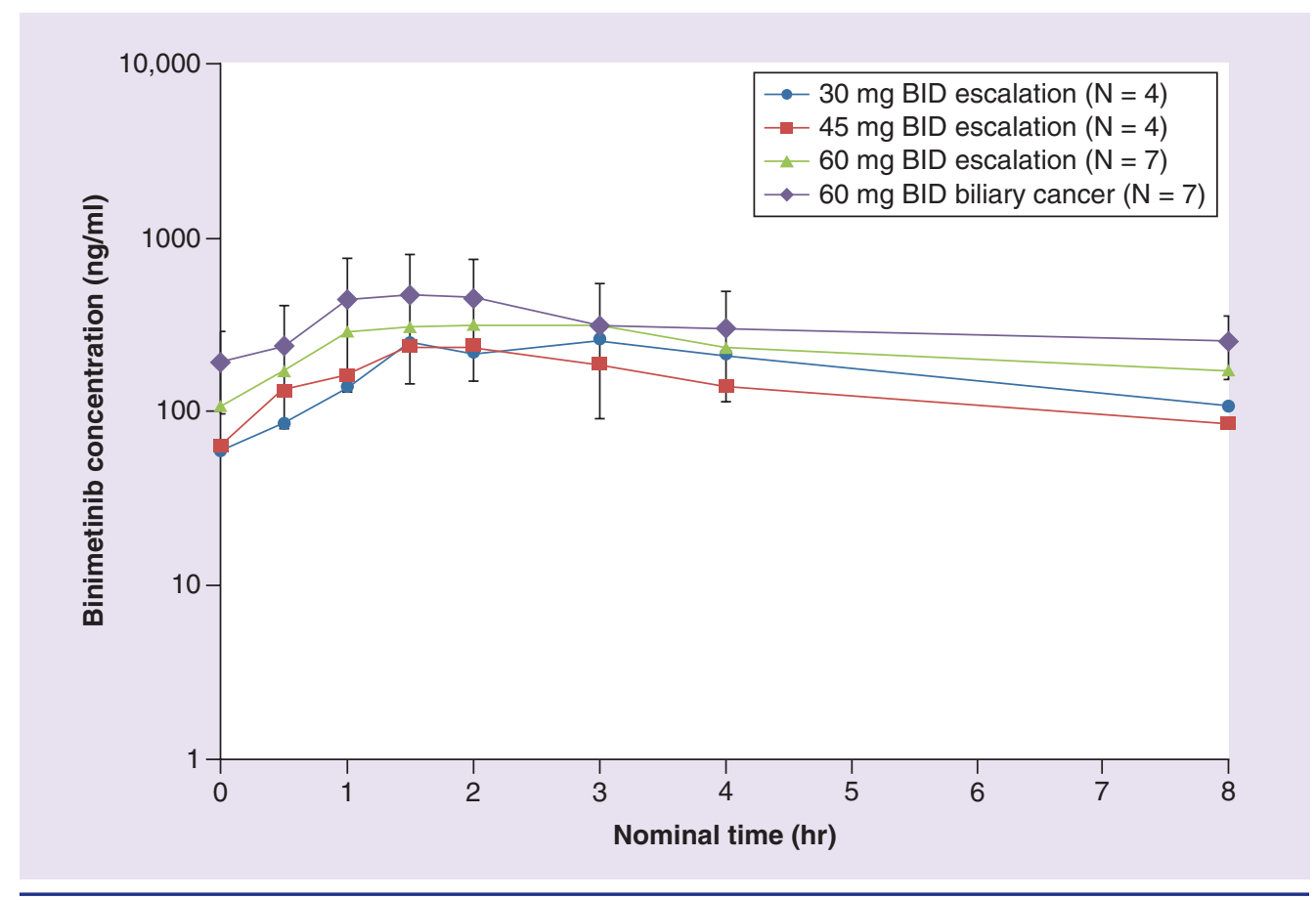

Figure 2. Geometric mean plasma concentrations of different binimetinib doses in Phase I studies on day 15 , cycle 1.

b.i.d.: Two-times a day.

CYP2C19 combined are estimated to account for $17.8 \%$ of the clearance of binimetinib via the $\mathrm{N}$-demethylation metabolic pathway. The maximum contribution of direct glucuronidation to the clearance of binimetinib was estimated at $62.2 \%$.

\section{- Elimination}

It was shown that after a single oral dose of 45 $\mathrm{mg}$ of ${ }^{14} \mathrm{C}$-binimetinib in humans, a mean of $62.3 \%$ of the radioactivity dose was eliminated in the feces, while $31.4 \%$ was eliminated in the urine. A total of $6.5 \%$ of the radioactivity dose was excreted as binimetinib in the urine. The estimated mean renal clearance value of $1.78 \mathrm{l} / \mathrm{h}$ accounted for $6.3 \%$ of the total clearance value of $28.2 \mathrm{l} / \mathrm{h}[19]$.

\section{- Phase I \& II data}

Following preclinical studies that demonstrated activity of binimetinib in different tumor xenografts regardless of BRAF and RAS mutational status [20], the first study of binimetinib (MEK162) demonstrating tolerability and signs of clinical activity in humans was presented by Bendell et al. in 2011 [21]. An update of this study including two expansion cohorts with biliary and colorectal cancer patients was published very recently [22]. Initially, this group investigated binimetinib at increasing oral doses from 30 to $80 \mathrm{mg}$ twice daily in 19 predominantly male patients with different cancer types (colorectal, pancreatic, cholangiocarcinoma and others) harboring a heterogeneous mutation profile [21]. Dose-limiting toxicities occurred in two out of the four patients in the $80 \mathrm{mg}$ b.i.d. arm. These were a therapy-resistant acneiform rash and a central serous-like retinopathy, respectively. Of note, these toxicities resolved with interruption of dosing and re-challenge with a lower dose of binimetinib. Other frequent treatment-related adverse events (AEs) included gastrointestinal (diarrhea, nausea) and skin disorder as well as peripheral edema, increased creatine kinase and retinal disorders. Concerning clinical activity, one partial response (PR) out of the first 17 patients with efficacy-evaluable disease was reported in a patient with NRASQ61-mutated cholangiocarcinoma. Subsequently, the first expansion cohort of this study was initiated with the maximum tolerated dose of binimetinib (60 mg b.i.d.) in 28 patients with advanced biliary tract cancer and reported on in 2012 [23]. Due to a higher than expected rate of ocular toxicities - mainly central serous-like retinopathy - and dose reductions in this cohort, further 
expansion-phase patients were treated with the $45 \mathrm{mg}$ b.i.d. dose of binimetinib. Overall, out of 91 evaluable patients in this Phase I study, 3 patients with biliary cancer showed an objective response to binimetinib therapy (1 complete response [CR], 2 PRs). Notably, only one of the tumor samples was tested positive for an NRAS mutation, both other tumors responding to binimetinib treatment were wild-type for BRAF, NRAS and KRAS [22].

Based on the Phase I results described above and on the observation that MEK inhibition leads to dose-dependent growth suppression of BRAF- and NRAS-mutant melanoma xenografts [24], the first larger scale open-label Phase II clinical trial with binimetinib was conducted in patients with advanced melanoma [18]. This multicenter study, reported by Ascierto et al., was initially designed with three treatment arms. In two arms (BRAF-vs NRAS-mutated tumors) patients were enrolled to receive $45 \mathrm{mg}$ b.i.d. of binimetinib, while in the third arm patients with BRAF-mutated melanoma were planned to receive $60 \mathrm{mg}$ of binimetinib b.i.d.. The first published analysis of this study only reported on the two $45 \mathrm{mg}$ arms and will be discussed now in detail. A total of 41 and 30 patients with advanced melanoma harboring BRAF and NRAS mutations, respectively, were included in this analysis. Patients were mostly pretreated, with $66 \%$ of patients in the BRAF and $77 \%$ in the NRAS group having received at least one line of prior anticancer therapy. The primary end point of the study was ORR and was reached in $20 \%$ of patients in both groups according to investigator assessment ( 8 out of 41 BRAF-mutated and 6 out of 30 NRAS-mutated tumors responded). After central review, PRs were confirmed in three patients $(10 \%)$ with NRAS-mutated and in two patients (5\%) with BRAF-mutated melanoma. After a median follow-up (FU) of 3.3 months (14 weeks), the median DOR was 7.6 weeks (0.1-17.3) and 9.2 weeks $(0.1-16.1)$ in the NRAS- and BRAF-mutated group, respectively. The types of treatment-related AEs were similar to those reported in earlier binimetinib studies and did not differ significantly between the BRAF- and the NRAS-mutated treatment arms. Serious AEs (common toxicity criteria [CTC] grade 3 or 4 ) occurred in 4 out 71 patients $(5.6 \%)$ in total. 15 patients $(21 \%)$ discontinued treatment due to AEs and 33 patients $(46 \%)$ had at least one dose reduction after occurrence of an $\mathrm{AE}$.
Results on an expanded cohort of this trial with 117 NRAS-mutated melanoma patients were presented by Van Herpen et al. in 2014 [25]. The confirmed RR in these patients was $14.5 \%$ with a median progression-free survival (PFS).

of 3.6 (2.6-3.8) months. The AE profile of binimetinib in this extended cohort remained relatively unchanged.

In summary, these results were the first to prospectively confirm the clinical activity of an MEK inhibitor in advanced NRAS-mutated melanoma and led to the initiation of the randomized Phase III study outlined next, the socalled NEMO trial.

\section{- Phase III data \\ NEMO Study}

The Phase III randomized open-label, multicenter NEMO (NRAS melanoma and MEK inhibitor) trial comparing binimetinib $45 \mathrm{mg}$ b.i.d. with dacarbazine (DTIC) $1000 \mathrm{mg} / \mathrm{m}^{2}$ intravenously $\mathrm{q} .3 \mathrm{w}$. was conducted in patients with advanced unresectable or metastatic melanoma harboring an NRAS mutation [26]. Stratification factors included performance status, tumor stage and prior immunotherapy. Between July 2013 and April 2015, 402 patients were enrolled and randomized in a 2:1 ratio (269 in the binimetinib and 133 in the DTIC arm).

The primary end point of the study was PFS per blinded central review and was reached with binimetinib showing superior median PFS as compared with DTIC (2.8 [2.8-3.6] vs 1.5 months [1.5-1.7], hazard ratio [HR]: 0.62; 95\% CI: 0.47-0.80; one-sided p-value $<0.001$ ) (Figure 3). The median FU for this analysis was 1.7 months (interquartile range [IQR] 1.4-4.1). Median OS was 11.0 months (8.9-13.6) in the binimetinib and 10.1 months (7.0-16.5) in the DTIC arm (HR: 1.00 [0.75-1.33]; p = 0.499 ) after a median FU of 9.2 months (IQR 4.8-13.9; Figure 3). Confirmed ORR was significantly higher in binimetinib-treated compared with DTIC-treated patients $(15.2 \%$ [95\% CI: $11.2-20.1 \%$; $\mathrm{n}=41]$ vs $6.8 \%$ [95\% CI: $3.1-$ $12.5 \% ; n=9] ; p=0.015$, two-sided test). Four patients $(1.5 \%)$ in the binimetinib arm achieved a CR versus zero patients in the DTIC arm. The median DOR was 6.9 months for binimetinib and not evaluable for DTIC. Disease control could be achieved in 58.4\% ( $n=157 / 269)$ of patients treated with binimetinib and in $24.8 \%$ $(n=33 / 133)$ of DTIC-treated patients. 
DRUG EVALUATION Koelblinger, Dornbierer \& Dummer

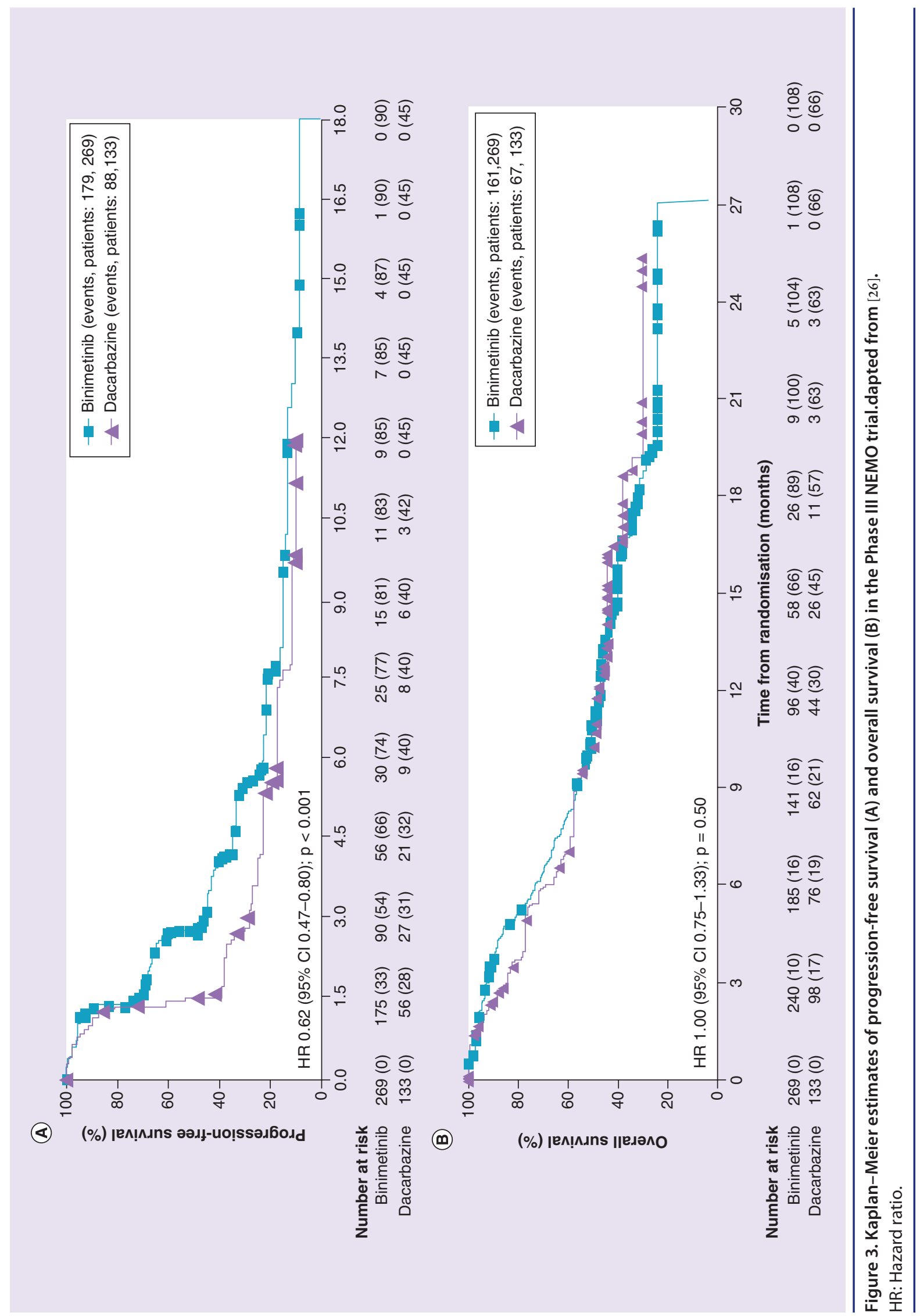


PFS was consistent with the overall population in most predefined subgroups such as in patients with elevated lactate dehydrogenase or M1c disease at baseline. Of note, the subgroup of patients who received prior immunotherapy for advanced disease $(n=85)$ showed longer PFS when treated with binimetinib in comparison with DTIC (5.5 months [95\% CI: 2.8-7.6] vs 1.6 months [95\% CI: 1.5-2.8]). Correspondingly, the median DOR was 11.1 months in binimetinib patients pretreated with immunotherapy versus 6.9 months in the overall binimetinib-treated patient population. The confirmed ORR for binimetinib in this subgroup was in turn comparable to the overall population (15.8 vs $15.2 \%$ ).

Serious AEs due to binimetinib treatment occurred at a frequency of $33.8 \%(n=91 / 269)$ in this study, which is higher than reported in Phase I and II trials with binimetinib. A total of $24.5 \%$ ( $n=66 / 269)$ of all binimetinib-treated patients had to discontinue treatment due to AEs. AEs resulting in dose reduction occurred in $60.6 \%$ ( $n=163 / 269)$ of binimetinib patients. The most frequent AEs leading to discontinuation of binimetinib treatment were decreased ejection fraction $(3.7 \%$ [ $n=10 / 269])$, increased creatine phosphokinase (CPK) $(1.9 \%[\mathrm{n}=5 / 269])$, retinal vein occlusion $(1.9 \%[n=5 / 269])$ and retinal detachment $(1.5 \%[n=4 / 269])$. Regardless of CTC grade, the most frequent AEs included increased CPK (42\%), diarrhea (40\%), rash $(36 \%)$, peripheral edema $(36 \%)$, acneiform dermatitis (35\%), nausea (29\%), fatigue (22\%) and vomiting $(21.2 \%)$. Ocular toxicities of binimetinib were mostly summarized as retinal pigment epithelial detachment occurring at an overall frequency of $14.5 \%$ and were reported to be generally self-limiting and reversible after dose modification. Latter is not the case for the rare occurrence of retinal vein occlusion which led to permanent treatment discontinuation in all affected patients $(n=6 / 269 ; 2.2 \%)$. Other AEs of clinical interest were cardiac events, mostly asymptomatic decrease of ejection fraction, reported in $13 \%(n=35 / 269)$ and grade $3 / 4$ hypertension in $8.6 \%(n=23 / 269)$ of patients. One death due to multiorgan failure in the binimetinib arm was considered to be treatment related by the investigator.

\section{COLUMBUS study}

Besides the application as single-agent therapy in NRAS-mutated melanoma, binimetinib has also been investigated in combination with the BRAF inhibitor encorafenib in BRAF-mutated melanoma [27]. Encorafenib is an orally bioavailable, highly potent second-generation inhibitor of the V600-mutated BRAF protein and has shown efficacy and tolerability both in preclinical and early clinical studies in BRAF-mutated melanoma [28-30]. It has a long dissociation time which is at least six-times longer than that of other BRAF inhibitors. In November 2016, first results on the combined use of encorafenib and binimetinib in part 1 of the randomized Phase III COLUMBUS trial (Combined LGX818 Used with MEK162 in BRAF Mutant Unresectable Skin Cancer) were presented [27]. This trial compared the combination (combo) of encorafenib $450 \mathrm{mg}$ once a day (q.d.) and binimetinib $45 \mathrm{mg}$ b.i.d. with BRAF-inhibitor monotherapy with either encorafenib $300 \mathrm{mg}$ q.d. (enco) or vemurafenib $960 \mathrm{mg}$ b.i.d. (vem). Patients were randomized in one of the three treatment arms in a 1:1:1 fashion and stratified according to American Joint Committee on Cancer (AJCC) stage, Eastern Cooperative Oncology Group (ECOG) status, BRAF mutation subtype (V600 E or K) and prior first-line immunotherapy. A total of 577 patients with well-balanced baseline characteristic in the three treatment arms were enrolled into the study. Five percent of all patients had received prior treatment with a checkpoint inhibitor (ipilimumab or programmed death 1 [PD-1] antibody).

The primary end point of the study was median PFS which was shown to be significantly higher in the combination treatment arm as compared with the two monotherapy arms by central review (14.9 months [11.0-18.5] for combo vs 9.6 months [7.5-14.8] for enco vs 7.3 months [5.6-8.2] for vem therapy; HR: 0.54 [0.41-0.71] for combo vs vem $[\mathrm{p}=0.001]$ and 0.75 [0.56-1.00] for combo vs enco $[\mathrm{p}=0.051])$. Confirmed ORRs by central review were 63 (5670), 51 (43-58) and 40\% (33-48) for combo, enco and vem with a median DOR of 16.2, 14.8 and 8.4 months, respectively. Subgroup analysis showed a consistent PFS benefit of patients treated with the combination therapy in most predefined subgroups, especially when compared with vemurafenib.

Grade 3/4 AEs were slightly less frequent in the combo arm occurring in $58 \%$ of patients, compared with 66 and $63 \%$ in the enco and vem arm, respectively. $8 \%$ of combo patients discontinued treatment due to AEs, while 12 and $14 \%$ 
of enco and vem patients did so. As known from other combination therapy studies [4-6], typical BRAFinhibitor related AEs, such as arthralgias or hyperkeratosis occurred less frequent through addition of the MEK inhibitor. Conversely, typical MEK inhibitor associated toxicities, such as CPK increase or ocular toxicities were almost solely reported in the combo arm, albeit less frequent than with binimetinib monotherapy in the NEMO study.

The differences between the combo and enco arm concerning PFS and other efficacy parameters reported in this first analysis are difficult to interpret, as a lower encorafenib dose was used in the monotherapy arm. The primary objective of the ongoing part 2 of the COLUMBUS trial is to further evaluate the contribution of binimetinib to combination therapy by comparing an equal dose of encorafenib (300 mg q.d.) as monotherapy and in combination with binimetinib.

\section{Discussion}

Binimetinib is both the first MEK inhibitor and the first small molecule that has shown clinical activity in advanced NRAS-mutant melanoma in prospective Phase II and III clinical trials. Although the reported RR of $15 \%$ in the Phase III NEMO study is modest and not comparable to the RRs of $60-70 \%$, we have been accustomed to the combined targeted therapy in BRAF-mutated melanoma, and binimetinib may now be considered as an alternative treatment to palliative chemotherapy after progression to immunotherapy in patients with NRASmutated tumors.

As suggested by a subgroup analysis of the NEMO study, patients with prior immunotherapy with an anti-CTLA-4 or anti-PD-1 antibody may even experience more durable responses to binimetinib, albeit the ORR did not differ significantly in this subgroup of patients. Previously, due to the observation of increased RRs to high-dose IL-2 in NRASmutated patients, it has been proposed that NRAS-mutated tumors may be more susceptible to immunotherapy in general [31]. More recently, however, this hypothesis could not be confirmed for the anti-CTLA-4 antibody ipilimumab in an analysis lead by our institution [32]. Yet, the rational for sequential or concurrent use of immunotherapy and MEK inhibitors is also supported by preclinical data. Ebert et al. showed that combined MEK- and PD-ligand 1 markedly enhances tumor response in mice with KRAS-mutated colon cancer xenografts [33]. These authors also described an immunotherapeutic effect of MEK inhibitors per se by demonstrating an increase in the number of intratumoral antigen-specific $\mathrm{CD}^{+}$effector $\mathrm{T}$ cells after MEK inhibition. These preclinical findings have already been translated into melanoma patients in a recently presented Phase Ib study [34]. The promising results of this study in turn led to the design of a Phase III clinical trial comparing the combination of the MEK inhibitor cobimetinib and the anti-PD-ligand 1 antibody atezolizumab with anti-PD-1 antibody monotherapy in BRAF wild-type melanoma.

Apart from binimetinib, several other MEK inhibitors have been analyzed in NRAS-mutated melanoma: Subanalyses of Phase I and II trials did not report responses for the MEK inhibitors trametinib and selumetinib in nine and ten patients with NRAS-mutated tumors, respectively $[35,36]$. In contrast, clinical activity of pimasertib wasshown recently in a multicenter, openlabel Phase 2 trial with 194 patients showing a response rate of $23 \%$ and a significant improvement in PFS as compared to DTIC chemotherapy (median PFS: 13.0 vs 6.9 months; $\mathrm{p}=$ 0.0022 ) [37]. Of note, in BRAF-mutated melanoma, the efficacy of trametinib monotherapy with an ORR of $22 \%$ and a median PFS of 4.8 months, as reported in the Phase III METRIC trial, seems to be superior when compared with the Phase II data for binimetinib in BRAFmutated tumors outlined earlier $[18,38]$.

Overall, safety and tolerability of monotherapy with binimetinib in advanced melanoma can be considered acceptable. AEs typically include rash, acneiform dermatitis, diarrhea, fatigue, hypertension, central serous-like retinopathy and peripheral edema. These class-specific side effects have also been described at a similar frequency with other MEK inhibitors [38]. However, certain AEs, in particular an increase of blood CPK, occur at a higher frequency with binimetinib and contribute to the markedly elevated rate of dose reductions and treatment discontinuations reported in the NEMO trial in comparison with Phase III data for trametinib in the METRIC study $[26,38]$.

In advanced BRAF V600-mutated melanoma, combination targeted therapy with either dabrafenib plus trametinib $(\mathrm{D}+\mathrm{T})$ or vemurafenib plus cobimetinib $(\mathrm{V}+\mathrm{C})$ is currently considered standard of care aside from 
immunotherapy. The results of the Phase III COLUMBUS trial summarized above show that the combination of encorafenib and binimetinib seems to be at least as efficient as the established targeted therapy combinations in this patient population. The confirmed ORR ( $63 \%$ by central and $75 \%$ by local review) and median PFS of 14.9 months in patients treated with encorafenib plus binimetinib compare well to the numbers reported in PhaseIII studies of the other combination regimens $(\mathrm{D}+\mathrm{T}$ : ORR 64-69\%, PFS 11.0-11.4 months, V+C: ORR 69.6\%, PFS 12.3 months) [39]. OS data for encorafenib plus binimetinib are expected to be presented in the course of 2017 and will further shed light on the role to be taken by this new combination therapy. Safety and tolerability of the encorafenib plus binimetinib regimen appear favorable to the established alternatives because there are few fever reactions and little or no phototoxicity. These frequent side effects are considered the clinically most relevant of $\mathrm{D}+\mathrm{T}$ and $\mathrm{V}+\mathrm{C}$, respectively.

\section{Regulatory affairs}

Based on the results of the NEMO trial, the use of binimetinib as a monotherapy in advanced NRAS-mutated melanoma is currently under review both by the US FDA and the by the EMA in Europe. Most recently, however, the manufacturer of binimetinib has withdrawn its FDA new drug application for binimetinib monotherapy in this indication due to feedback from the agency.

\section{Table 1. Overview of clinical studies of binimetinib.}

\begin{tabular}{|c|c|c|c|c|}
\hline Study (year) & Study design & Efficacy findings & Safety findings & Ref. \\
\hline $\begin{array}{l}\text { Finn et al. } \\
(2012)\end{array}$ & $\begin{array}{l}\text { Phase I expansion } \\
\text { cohort with BINI } \\
60 \text { mg b.i.d.; } \mathrm{n}=28 \\
\text { (biliary tract cancer) }\end{array}$ & $\begin{array}{l}\text { - RR: } 8 \% \text { ( } 2 \text { of } 26 \text { pts); } 1 \text { PR, } 1 \text { CR } \\
\text { - SD } \geq 6 \text { weeks in } 12 \text { pts }(46 \%)\end{array}$ & $\begin{array}{l}\text { Frequent any grade AEs: rash (78\%), nausea (43\%), } \\
\text { vomiting (36\%), peripheral edema (32\%), diarrhea } \\
(32 \%), \text { fatigue ( } 29 \%) \text { and retinal toxicity }(21 \%) \\
\text { Dose reductions due to AEs in } 13 \text { pts }(46 \%)\end{array}$ & {$[23]$} \\
\hline $\begin{array}{l}\text { Ascierto et al. } \\
(2013)\end{array}$ & $\begin{array}{l}\text { Phase II multicenter } \\
\text { open-label, BINI } 45 \\
\text { mg b.i.d. in NRAS- ( } \mathrm{n} \\
=30 \text { ) and BRAF- } \\
\text { mutated melanoma } \\
(\mathrm{n}=41)\end{array}$ & $\begin{array}{l}\text { - Investigator-assessed RR of } 20 \% \text { in } \\
\text { NRAS- and BRAF-mutated ( } 6 / 30 \text { and } 8 / 41 \\
\text { pts) } \\
\text { - Confirmed PRs in } 3 \text { and } 2 \text { pts only, no } \\
\text { CRs } \\
\text { - SD in } 13 \text { (42\%) NRAS-mut. and } 13 \text { (32\%) } \\
\text { BRAF-mut. pts } \\
\text { Survival: } \\
\text { - Median PFS for NRAS-mut.: } 3.7 \text { mo } \\
\text { (95\% Cl: } 2.5-5.4) \\
\text {-Median PFS for BRAF-mut.: } 3.6 \text { mo ( } 95 \% \\
\text { Cl: } 2.0-3.8)\end{array}$ & $\begin{array}{l}\text { Frequent any grade AEs (NRAS- + BRAF-mut.; } n= \\
71) \text { : } \\
\text { Acneiform dermatitis ( } 46 \%) \text {, peripheral edema } \\
(34 \%) \text {, diarrhea ( } 32 \%) \text {, increased CPK }(28 \%) \text {, retinal } \\
\text { toxicity ( } 18 \%) \\
\text { Grade } 3 / 4: 4 \text { pts (5.6\%) } \\
\text { Treatment discontinuation due to AEs: } 15 \text { pts ( } 21 \%) \\
\text { Dose reduction due to AEs: } 33 \text { pts }(46 \%)\end{array}$ & {$[18]$} \\
\hline $\begin{array}{l}\text { Dummer et al. } \\
\text { (2017) } \\
\text { NEMO }\end{array}$ & $\begin{array}{l}\text { Phase III multicenter } \\
\text { open-label, } 2: 1 \\
\text { randomization: BINI } \\
45 \text { mg b.i.d. }(\mathrm{n}=269) \\
\text { vs DTIC } 1000 \mathrm{mg} / \mathrm{m}^{2} \\
\text { iv. q.3w. }(\mathrm{n}=133) \\
\text { NRAS-mutated } \\
\text { melanoma }\end{array}$ & $\begin{array}{l}\text { - Confirmed RR of } 15.2 \% \text { for BINI }(95 \% \\
\text { Cl: } 11.2-20.1 \%) \text { vs } 6.8 \% \text { for DTIC ( } 95 \% \\
\text { CI: } 3.1-12.5 \%) ; p=0.015 \\
\text { - SD: } 40.5 \% \text { (BINI) vs } 17.3 \% \text { (DTIC) } \\
\text { Survival: } \\
\text { - Median PFS: } 2.8 \text { months ( } 95 \% \mathrm{CI}: 2.8- \\
\text { 3.6) for BINI vs } 1.5 \text { months }(95 \% \mathrm{Cl}: 1.5- \\
\text { 1.7) for DTIC (HR: } 0.62 ; \mathrm{p}<0.001)\end{array}$ & $\begin{array}{l}\text { Frequent any grade AEs (BINI): increased CPK (42\%), } \\
\text { diarrhea (40\%), peripheral edema (36\%), rash (36\%), } \\
\text { acneiform dermatitis ( } 35 \%) \text {, retinal toxicity }(17 \%) \text {. } \\
\text { Serious AEs: } 91 \text { pts ( } 33.8 \%) \text { treatment } \\
\text { discontinuation due to AEs: } 66 \text { pts }(24.5 \%) \\
\text { Dose reduction due to AEs: } 163 \text { pts }(60.6 \%)\end{array}$ & [26] \\
\hline $\begin{array}{l}\text { Dummer et al. } \\
(2016) \\
\text { COLUMBUS }\end{array}$ & $\begin{array}{l}\text { Phase III multicenter } \\
\text { open-label, 1:1:1 } \\
\text { randomization ( } \mathrm{n}= \\
\text { 577): ENCO } 450 \mathrm{mg} \\
\text { q.i.d. + BINI } 45 \mathrm{mg} \\
\text { b.i.d. (Combo) vs VEM } \\
960 \text { mg BID vs ENCO } \\
300 \text { mg q.i.d. } \\
\text { BRAF-mutated } \\
\text { melanoma }\end{array}$ & $\begin{array}{l}\text { Combo vs VEM vs ENCO } \\
\text { Confirmed RR: } \\
-63 \%(56-70) \text { vs } 40 \%(33-48) \text { vs } 51 \% \\
(43-58) \\
\text { Survival: } \\
\text { - Median PFS: } 14.9 \text { months ( } 11.0-18.5) \\
\text { vs } 7.3 \text { months }(5.6-8.2) \text { vs } 9.6 \text { months } \\
\text { (7.5-14.8) HR: } 0.54 \text { for Combo vs vem ( } p \\
=0.001) \text { and } 0.75 \text { for combo vs enco ( } p \\
=0.051)\end{array}$ & $\begin{array}{l}\text { Frequent any grade AEs (combo only): nausea } \\
(41 \%) \text {, diarrhea ( } 36 \%) \text {, vomiting ( } 30 \%) \text {, fatigue ( } 29 \%) \text {, } \\
\text { arthralgia ( } 26 \%) \text {, increased CPK ( } 23 \%) \text {, headache } \\
(22 \%) \text {, pyrexia (18\%), retinal toxicity (13\%) } \\
\text { Grade } 3 / 4 \text { AEs: } 58 \% \text { of pts } \\
\text { Treatment discontinuation due to AEs : } 16 \text { pts ( } 8 \%) \\
\text { Dose reduction due to AEs: } 21 \text { pts (11\%) } \\
\text { Dose interruption due to AEs: } 88 \text { pts }(46 \%)\end{array}$ & {$[27]$} \\
\hline
\end{tabular}


Conclusion

The MEK inhibitor binimetinib has been shown to improve PFS and RRs compared with DTIC chemotherapy in patients with NRAS-mutated melanoma and, regardless of approval status, can be considered as a treatment alternative to palliative chemotherapy in this patient subgroup after failure to immunotherapy. Moreover, the combination of binimetinib with the BRAF inhibitor encorafenib has yielded promising results regarding treatment efficacy and tolerability in BRAF-mutated melanoma. Once OS data for this novel BRAF-/MEK-I combination have been published, it may emerge as an alternative to existing approved combination regimens.

Table 1
Financial \& competing interests disclosure

$P$ Koelblinger has received honoraria for travel support and consulting/advisory roles for Roche, Bristol Myers Squibb (BMS), Merck Sharp \& Dome (MSD), Novartis, and Amgen outside the submitted work. R Dummer has intermittent project focused consulting and/or advisory relationships with Novartis, MSD, BMS, Roche, Amgen, Takeda, and Pierre Fabre outside the submitted work. The authors have no other relevant affiliations or financial involvement with any organization or entity with a financial interest in or financial conflict with the subject matter or materials discussed in the manuscript apart from those disclosed.

No writing assistance was utilized in the production of this manuscript.

\section{EXECUTIVE SUMMARY}

\section{Binimetinib pharmacokinetic properties}

- Binimetinib is rapidly absorbed after oral administration and mainly eliminated via feces. Concurrent food intake delays the absorption of binimetinib but does not influence the total dose absorbed.

\section{Phase I evidence for binimetinib}

- Multiple Phase I clinical studies have evaluated binimetinib in different cancer types. Clinical activity was observed in biliary cancer and melanoma. No responses were reported in a Phase I expansion cohort with colorectal cancer patients. A total of $45 \mathrm{mg}$ of binimetinib two-times a day was defined as the recommended dose for Phase II trials.

\section{Phase Il data of binimetinib in BRAF- or NRAS-mutated melanoma}

- A Phase Il study in 30 NRAS- and 41-BRAF-mutated melanoma patients showed a confirmed response rate (RR) of 10 and $5 \%$, respectively. Disease stabilization was reported in about a third of patients. The RR improved to nearly $15 \%$ in an expansion cohort with 117 NRAS-mutated melanoma patients.

Phase III clinical trials with binimetinib as single agent or combination therapy

- The NEMO trial compared binimetinib $45 \mathrm{mg}$ b.i.d. with dacarbazine chemotherapy in almost 400 patients with NRASmutated melanoma. The confirmed RR of $15.2 \%$ with binimetinib was significantly higher than the $6.8 \%$ reported for dacarbazine. Also, PFS was significantly increased after binimetinib treatment (2.8 vs 1.5 months). No significant overall survival (OS) difference was noted. The most common adverse events reported were in line with Phase I and II studies and included increased creatine phosphokinase, diarrhea, peripheral edema, rash, acneiform dermatitis and retinal toxicity.

- The three-arm COLUMBUS trial compared the combination of binimetinib and encorafenib with BRAF-inhibitor monotherapy with either encorafenib or vemurafenib in 577 BRAF-mutated melanoma patients. The RR of $63 \%$ and median PFS of 14.9 months reported for the combination were superior to BRAF-inhibitor monotherapy. OS data have not been reported. Tolerability of binimetinib seems to be better when combined with encorafenib as compared with monotherapy.

\section{Conclusion}

- Although no OS benefit has been observed in the NEMO trial, binimetinib is the first targeted therapy shown to improve PFS in NRAS-mutated melanoma patients and may be considered as an alternative treatment to chemotherapy in NRAS-mutated melanoma patients who progress after initial immunotherapy. Combination regimens of binimetinib with immunotherapy should be explored based on preclinical and early clinical data.

- Results of the combination of binimetinib with encorafenib in BRAF-mutated melanoma are promising both concerning treatment efficacy and tolerability. First OS data are expected to be presented in the course of 2017 and will further define the role of this novel combination in the field of targeted therapy. 


\section{References}

Papers of special note have been highlighted as: • of interest; $\bullet$ of considerable interest

1 Chapman PB, Hauschild A, Robert C et al. Improved survival with vemurafenib in melanoma with BRAF V600E mutation. $N$. Engl. J. Med. 364 (26), 2507-2516 (2011).

- Vemurafenib improved progression-free survival (PFS) and overall survival (OS) as compared with dacarbazine (median PFS: 5.3 vs 1.6 months, hazard ratio [HR] for death in the vemurafenib group was 0.37 [95\% CI: 0.26-0.55; $\mathrm{p}<0.001]$ ).

2 Flaherty KT, Infante JR, Daud A et al. Combined BRAF and MEK inhibition in melanoma with BRAF V600 mutations. $N$. Engl. J. Med. 367(18), 1694-1703 (2012).

3 Hauschild A, Grob JJ, Demidov LV et al. Dabrafenib in BRAF-mutated metastatic melanoma: a multicentre, open-label, Phase III randomised controlled trial. Lancet 380 (9839), 358-365 (2012).

4 Larkin J, Ascierto PA, Dreno B et al. Combined vemurafenib and cobimetinib in BRAF-mutated melanoma. N. Engl. J. Med. 371(20), 1867-1876 (2014).

- Combination of vemurafenib and cobimetinib improved response rate (68 vs 45\%; p < 0.001) and median PFS (9.9 vs 6.2 months, HR for death or disease progression: 0.51 ; 95\% CI: 0.39-0.68; $\mathrm{p}<$ 0.001) compared with vemurafenib monotherapy.

5 Long GV, Stroyakovskiy D, Gogas $\mathrm{H}$ et al. Dabrafenib and trametinib versus dabrafenib and placebo for Val600 BRAF-mutant melanoma: a multicentre, double-blind, Phase III randomised controlled trial. Lancet 386(9992), 444-451 (2015).

- The combination of dabrafenib and trametinib increased response rate, PFS and OS compared with dabrafenib alone (median OS: 25.1 months [95\% CI: 19.2not reached] vs 18.7 months [95\% CI: 15.2-23.7]; HR: 0.71 [95\% CI: 0.55-0.92; p $=0.011$.

6 Robert C, Karaszewska B, Schachter J et al. Improved overall survival in melanoma with combined dabrafenib and trametinib. $N$. Engl. J. Med. 372(1), 30-39 (2015).

7 Schadendorf D, Hodi FS, Robert C et al. Pooled analysis of long-term survival data from Phase II and Phase III trials of ipilimumab in unresectable or metastatic melanoma. J. Clin. Oncol. 33(17), 1889-1894 (2015).

8 Cancer Genome Atlas Network. Genomic classification of cutaneous melanoma. Cell 161(7), 1681-1696 (2015).

9 Krauthammer M, Kong Y, Bacchiocchi A et al. Exome sequencing identifies recurrent mutations in NF1 and RASopathy genes in sun-exposed melanomas. Nat. Genet. 47(9), 996-1002 (2015).

10 Davies H, Bignell GR, Cox C et al. Mutations of the BRAF gene in human cancer. Nature 417(6892), 949-954 (2002).

11 Lee JH, Choi JW, Kim YS. Frequencies of BRAF and NRAS mutations are different in histological types and sites of origin of cutaneous melanoma: a meta-analysis. $\mathrm{Br}$. J. Dermatol. 164(4), 776-784 (2011).

12 Raaijmakers MI, Widmer DS, Narechania A et al. Co-existence of BRAF and NRAS driver mutations in the same melanoma cells results in heterogeneity of targeted therapy resistance. Oncotarget 7(47), 77163-77174 (2016).

13 Johnson DB, Menzies AM, Zimmer L et al. Acquired BRAF inhibitor resistance: a multicenter meta-analysis of the spectrum and frequencies, clinical behaviour, and phenotypic associations of resistance mechanisms. Eur. J. Cancer 51(18), 2792-2799 (2015).

14 Dummer R, Hauschild A, Lindenblatt N, Pentheroudakis G, Keilholz U, Committee EG. Cutaneous melanoma: ESMO Clinical Practice Guidelines for diagnosis, treatment and follow-up. Ann. Oncol. 26(Suppl. 5), v126-v132 (2015).

15 Garbe C, Peris K, Hauschild A et al. Diagnosis and treatment of melanoma. European consensus-based interdisciplinary guideline - update 2016. Eur. J. Cancer 63, 201-217 (2016).

16 Coit DG, Thompson JA, Algazi A et al. Melanoma, Version 2.2016, NCCN Clinical Practice Guidelines in Oncology. J. Natl Compr. Canc. Netw. 14(4), 450-473 (2016).

17 Posch C, Vujic I, Monshi B et al. Searching for the chokehold of NRAS mutant melanoma. J. Invest. Dermatol. 136(7), 1330-1336 (2016).

18 Ascierto PA, Schadendorf D, Berking C et al. MEK162 for patients with advanced melanoma harbouring NRAS or Val600 BRAF mutations: a non-randomised, open-label Phase II study. Lancet Oncol. 14(3), 249-256 (2013).

- First Phase II study showing clinical activity of binimetinib in melanoma. Six out of 30 patients (20\%) with NRAS-mutated melanoma had a partial response $(3$ confirmed) and 8 out of 41 patients (20\%) with BRAF-mutated melanoma (two confirmed).

19 Array Biopharma Inc. Investigator's Brochure Binimetinib (MEK162), Edition 12.1. (2016).

20 Winski S, Anderson D, Bouhana K et al. MEK162 (ARRY-162), a Novel MEK 1/2 Inhibitor, Inhibits Tumor Growth Regardless of KRas/Raf Pathway Mutations. Presented at: 22nd EORTC-NCI-AACR symposium on Molecular targets and Cancer Therapeutics. Berlin, Germany, 27 May 2010.

21 Bendell J, Papadopoulos K, Jones S et al. A Phase I Dose-Escalation Study of MEK Inhibitor MEK162 (ARRY-438162) in Patients with Advanced Solid Tumors. Presented at: 2011 AACR-NCI-EORTC International Conference: Molecular Targets and Cancer Therapeutics. San Francisco, CA, USA, 12-16 November 2011.

22 Bendell JC, Javle M, Bekaii-Saab TS et al. A Phase I dose-escalation and expansion study of binimetinib (MEK162), a potent and selective oral MEK1/2 inhibitor. Br. J. Cancer 116(5), 575-583 (2017).

- Large Phase I study of binimetinib with two expansion cohorts (biliary and colorectal cancer) defining the Phase II dose of binimetinib as $45 \mathrm{mg}$ two-times a day. Clinical activity of binimetinib was seen in biliary cancer and melanoma.

23 Finn RS, Javle MM, Tan BR et al. A Phase I Study of MEK Inhibitor MEK162 (ARRY438162) in Patients with Biliary Tract Cancer. Presented at: 2012 ASCO Annual Meeting, Chicago, IL, USA, 1-5 June 2012.

24 Solit DB, Garraway LA, Pratilas CA et al. BRAF mutation predicts sensitivity to MEK inhibition. Nature 439 (7074), 358-362 (2006).

25 Van Herpen CM, Agarwala SS, Hauschild A et al. Overall Survival and Biomarker Results From a Phase II Study of MEK1/2 Inhibitor Binimetinib (MEK162) in Patients With Advanced NRAS-Mutant Melanoma. Presented at: European Society for Medical Oncology (ESMO) 2014 Congress. Madrid, Spain, 26-30 September 2014.

26 Dummer R, Schadendorf D, Ascierto PA et al. Binimetinib versus dacarbazine in patients with advanced NRAS-mutant melanoma (NEMO): a multicentre, open-label, randomised, Phase III trial. Lancet Oncol. 18(4), 435-445 (2017).

- Binimetinib significantly improved PFS in NRAS mutated melanoma compared with dacarbazine (Median PFS: 2.8 (95\% CI: 2.8-3.6) versus 1.5 months (95\% CI: 1.5-1.7), HR 0.62 (95\% CI: 0.47-0.80) 
one-sided $\mathrm{p}<0.001$ ).

27 Dummer R, Ascierto PA, Gogas HJ et al. Results of COLUMBUS Part 1: A Phase III Trial of Encorafenib (ENCO) Plus Binimetinib (BINI) Versus Vemurafenib (VEM) or ENCO in BRAF-Mutant Melanoma. Presented at: Society for Melanoma Research Thirteenth International Congress. Boston, MA, USA, 6-9 November 2016.

-• Combination of encorafenib with binimetinib significantly prolonged PFS in comparison with encorafenib or vemurafenib monotherapy in BRAF-mutated melanoma (median PFS: 14.9 [95\% CI: 11.0-18.5] vs 9.6 [95\% CI: $7.5-14.8]$ vs 7.3 months $[95 \%$ CI: 5.6-8.2]).

28 Stuart DD, Li N, Poon DJ et al. Preclinical profile of LGX818: a potent and selective RAF kinase inhibitor [abstract]. Proceedings of the 103rd Annual Meeting of the American Association for Cancer Research 72 (Suppl. 8), 2012 (Abstract nr 3790).

29 Dummer R, Flaherty KT, Kefford R et al. The Development of Encorafenib (LGX818) and Binimetinib (MEK162) in Patients With Metastatic Melanoma. Presented at: $\mathrm{HemOnc}$ Today: Melanoma and Cutaneous Malignancies. New York, NY, USA, 11-12 April 2014.

30 Sullivan RJ, Weber JS, Patel SP et al. A Phase
$\mathrm{Ib} / \mathrm{II}$ study of BRAF inhibitor (BRAFi) encorafenib (ENCO) plus MEK inhibitor (MEKi) binimetinib (BINI) in cutaneous melanoma patients naive to BRAFi treatment. [abstract]. J. Clin. Oncol. 33(Suppl.), 2015 (Abstract 9007).

31 Joseph RW, Sullivan RJ, Harrell R et al. Correlation of NRAS mutations with clinical response to high-dose IL-2 in patients with advanced melanoma. J. Immunother. 35(1), 66-72 (2012).

32 Mangana J, Cheng PF, Schindler K et al. Analysis of BRAF and NRAS mutation status in advanced melanoma patients treated with anti-CTLA-4 antibodies: association with overall survival? PLoS ONE 10(10), e0139438 (2015).

33 Ebert PJ, Cheung J, Yang Y et al. MAP kinase inhibition promotes $\mathrm{T}$ cell and anti-tumor activity in combination with PD-L1 checkpoint blockade. Immunity 44(3), 609-621 (2016).

34 Infante J, Kim TM, Friedmann J et al. Safety and Clinical Activity of Atezolizumab Combined With Cobimetinib in Metastatic Melanoma. Society for Melanoma Research Thirteenth International Congress. Boston, MA, USA, 6-9 November 2016.

35 Falchook GS, Long GV, Kurzrock R et al. Dabrafenib in patients with melanoma, untreated brain metastases, and other solid tumours: a Phase I dose-escalation trial. Lancet 379 (9829), 1893-1901 (2012).

36 Kirkwood JM, Bastholt L, Robert C et al. Phase II, open-label, randomized trial of the MEK1/2 inhibitor selumetinib as monotherapy versus temozolomide in patients with advanced melanoma. Clin. Cancer Res. 18(2), 555-567 (2012).

37 Lebbe C, Dutriaux C, Lesimple T et al. Pimasertib versus dacarbazine in patients with cutaneous NRAS melanoma: a controlled, open-label Phase II trial with crossover. ESMO 2016 Congress. Annals of Oncology 276 379-400, Copenhagen (2016).

38 Flaherty KT, Robert C, Hersey P et al. Improved survival with MEK inhibition in BRAF-mutated melanoma. N. Engl. J. Med. 367(2), 107-114 (2012).

- Only Phase III study evaluating MEK inhibitor monotherapy in BRAF-mutated melanoma. Both median PFS (4.8 vs 1.5 months, HR for disease progression or death 0.45 [95\% CI: $0.33-0.63$ ], $\mathrm{p}<0.001$ ) and OS rate at 6 months ( 81 vs $67 \%$ despite crossover) were superior with trametinib compared with chemotherapy.

39 Eroglu Z, Ribas A. Combination therapy with BRAF and MEK inhibitors for melanoma: latest evidence and place in therapy. Ther. Adv. Med. Oncol. 8(1), 48-56 (2016). 